\title{
Improving the random encounter model method to estimate carnivore densities using data generated by conventional camera-trap design
}

\author{
Germán Garrote, Ramón Pérez de Ayala, Antón Álvarez, José M. Martín \\ Manuel Ruiz, Santiago de Lillo and Miguel A. Simón
}

\begin{abstract}
The random encounter model, a method for estimating animal density using camera traps without the need for individual recognition, has been developed over the past decade. A key assumption of this model is that cameras are placed randomly in relation to animal movements, requiring that cameras are not set only at sites thought to have high animal traffic. The aim of this study was to define a correction factor that allows the random encounter model to be applied in photo-trapping surveys in which cameras are placed along tracks to maximize capture probability. Our hypothesis was that applying such a correction factor would compensate for the different rates at which lynxes use tracks and the surrounding area, and should thus improve the estimates obtained with the random encounter model. We tested this using data from a wellknown Iberian lynx Lynx pardinus population. Firstly, we estimated Iberian lynx densities using a traditional cameratrapping design followed by spatially explicit capturerecapture analyses. We estimated the differential use rate for tracks vs the surrounding area using data from a lynx equipped with a GPS collar, and subsequently calculated the correction factor. As expected, the random encounter model overestimated densities by $378 \%$. However, the application of the correction factor improved the estimate and reduced the error to $16 \%$. Although there are limitations to the application of the correction factor, the corrected random encounter model shows potential for density estimation of species for which individual identification is not possible.
\end{abstract}

Keywords Camera traps, capture-recapture, carnivores, correction factor, density estimation, Iberian lynx, Lynx pardinus, random encounter model

GERMÁN GARROTE (Corresponding author, (1) orcid.org/0000-0002-6974-4513), José M. Martín, Manuel Ruiz and Santiago de Lillo Agencia de Medio Ambiente y Agua de Andalucía, c/ Johan Gutenberg s/n, Isla de la Cartuja, 41092 Seville, Spain. E-mail gergarrote@gmail.com

Ramón Pérez de Ayala World Wildlife Fund-Spain, Madrid, Spain

Antón Álvarez Instituto de Biología de la Conservación, Madrid, Spain

Miguel A. Simón Consejería de Medio Ambiente de la Junta de Andalucía, Jaén, Spain

Received 12 June 2018. Revision requested 9 August 2018

Accepted 20 December 2018. First published online 17 December 2019.

\section{Introduction}

T ethods that accurately estimate animal abundances or 1 densities can greatly enhance the ability to monitor and manage populations (Garrote et al., 2011; Zero et al., 2013). Camera traps are highly efficient in detecting elusive mammals such as carnivores (Cutler \& Swann, 1999) and over the last decade they have been used widely for estimating population sizes of secretive but individually recognizable animals (Foster \& Harmsen, 2012). Population sizes of the tiger Panthera tigris (Karanth \& Nichols, 1998), jaguar Panthera onca (Boron et al., 2016) and Iberian lynx Lynx pardinus (Garrote et al., 2011) have been estimated successfully using capture-recapture analyses of camera-trapping data. However, this method is of limited use for species that do not have markings that allow for the identification and enumeration of individuals (Villette et al., 2016).

As an alternative to capture-recapture analysis, photographic capture rates (photo captures per unit time) can be used as a density index for species that cannot be individually identified (Carbone et al., 2001). Nevertheless the use of camera-trapping rates as an index of abundance has been debated widely (Carbone et al., 2001; Jennelle et al., 2002; Karanth et al., 2003; O’Brien et al., 2003; Harmsen et al., 2009; Rovero et al., 2013). Although under certain circumstances camera-trap encounter indices may give accurate estimates of relative abundance (O'Brien et al., 2003), criticisms have noted that indices derived from camera-trap data vary between species, habitats, seasons and/or camera placements (Larrucea et al., 2007; Harmsen et al., 2009; Cusack, et al., 2015a,b; Mann et al., 2015). However, Rowcliffe et al. (2008) have developed a method for estimating animal density using camera traps without individual recognition. This method is based on modelling random encounters between animals and cameras and takes into account variables affecting the trapping rate. The probability that a camera detects an animal depends on the speed of the animal (distance travelled in a given time), the exposure time, the detection area of the camera and the number of animals present. The random encounter model method has been tested on only a few occasions against known densities derived by other methods (Rovero \& Marshall, 2009; Zero et al., 2013; Anile et al., 2014; Cusack et al., 2015a,b). Three studies have tested the effectiveness of the random encounter model as a method for estimating carnivore 
populations (wildcat Felis silvestris, Anile et al., 2014; lion Panthera leo, Cusack et al., 2015a,b; European pine marten Martes martes, Balestrieri et al., 2016). Overall, these studies concluded that the random encounter model is a promising method for obtaining density estimates and has the potential to be applied widely, although further field tests for different species and habitats are still needed.

A key assumption of the model is that cameras are placed randomly in relation to animal movements, sampling parts of the landscape in proportion to their coverage in the study area, which means that they should not be placed to either inflate or deflate encounter rates (Rowcliffe et al., 2013). Therefore, to avoid inflated encounter rates and exaggerated estimates, the random encounter model requires that cameras are not set only at sites thought to have high animal traffic (e.g. tracks, underpasses or water sources; Rowcliffe et al., 2013). Because of this requirement, rare species such as carnivores may be detected too infrequently for density estimates to be calculated (Rowcliffe et al., 2013). Many carnivore species are more likely to be detected along tracks than with random camera placements (Larrucea et al., 2007; Harmsen et al., 2009; Blake \& Mosquera, 2014; Di Bitetti et al., 2014; Cusack et al., 2015a,b). Even with camera traps set along roads, carnivore species have low detection rates because of their naturally low densities, large home ranges and secretive habits (Trolle \& Kéry, 2005; O'Connell \& Nichols, 2011). The random arrangement of cameras in relation to animal movements thus requires a much greater sampling effort to achieve detection rates that can be used to generate reliable estimates.

The aim of this study was to define a correction factor that enables the random encounter model method to be applied to traditional photo-trapping surveys of carnivores, with cameras placed on tracks to maximize capture probability. Our hypothesis was that if the differential use rate between tracks and the rest of the area is known, then it is possible to calculate a correction factor for expected deviations from random encounter model density estimates that are caused by the violation of the method's key assumption. We tested this hypothesis using data from a well-known Iberian lynx population, a species with fur patterns that allow identification of individual animals (Garrote et al., 2011). Firstly, we estimated Iberian lynx densities using spatially explicit capture-recapture. We then estimated the differential use rate for tracks vs surrounding area using GPS collar data, and calculated a correction factor. Finally, we compared the density estimates from the original random encounter model with those derived from the adjusted model, to evaluate the usefulness of the correction factor.

\section{Study area}

We conducted our study on a private estate in the Sierra de Andújar Natural Park, which lies within the known range of the Sierra Morena Iberian lynx population in south-eastern
Spain (Simón et al., 2012; Fig. 1). The area is managed for big game hunting and has high densities of red deer Cervus elaphus and wild boar Sus scrofa. Altitudes range between 200 and $1,500 \mathrm{~m}$, and the vegetation consists primarily of wellpreserved Mediterranean forests dominated by oaks Quercus ilex, Quercus faginea and Quercus suber, and scrublands with Quercus coccifera, Pistacia lentiscus, Arbutus unedo, Phillyrea angustifolia and Myrtus communis.

\section{Methods}

The estate collaborates with the long-term ( $>_{14}$ years) Iberian lynx conservation programme, which uses intensive camera trapping throughout the year to closely monitor individuals within the population (Gil-Sánchez et al., 2011). Data from continuously operating camera traps in the estate showed that 10 Iberian lynxes occupied the study area during the study period.

The camera-trap survey was carried out during September-October 2015 and followed traditional protocols used in Iberian lynx monitoring (Gil-Sánchez et al., 2011) that are designed to generate population estimates using capture-recapture analysis (Garrote et al., 2011). We placed the cameras on $2-3 \mathrm{~m}$ wide tracks that are built for use by cars moving around the hunting estates. Cameras were positioned in trees or on poles c. $50 \mathrm{~cm}$ above ground level and $5.5 \mathrm{~m}$ from the opposite side of the track. We discarded records of animals detected beyond this $5.5-\mathrm{m}$ limit to fix the detection radius required for the random encounter model analysis. We used nine Moultrie M-88o camera traps (PRADCO, Birmingham, USA) with a mean spacing of $1.49 \pm \mathrm{SE} 0.44 \mathrm{~km}$. Cameras operated continuously and the delay period between photographs was set at $30 \mathrm{~s}$. Every 15 days we checked all cameras to ensure they were working correctly and to download any photographs. No cameras malfunctioned and therefore there were no gaps in the data.

We considered the density derived from spatially explicit capture-recapture analysis as the baseline for comparison with the density estimates obtained using the random encounter model. Spatially explicit capture-recapture uses two distinct submodels within its workflow to compute densities $(D)$. One submodel simulates an animal's distribution from the capture history to give the individual's activity centre as an output, whereas the second simulates the capture process on the basis of the radial distance between the estimated centre of activity and the traps (Efford et al., 2009). Input data is in two files, one containing the name and geographical coordinates of the detectors (cameras) and another containing the capture histories (i.e. season, animal identification, the occasion and the detector). We set the trap detector type for the camera-trapping analysis as proximity (allowing for multiple detections of the same individual during the same event; Efford et al., 2009), the distribution model as Poisson (assuming homogeneous 


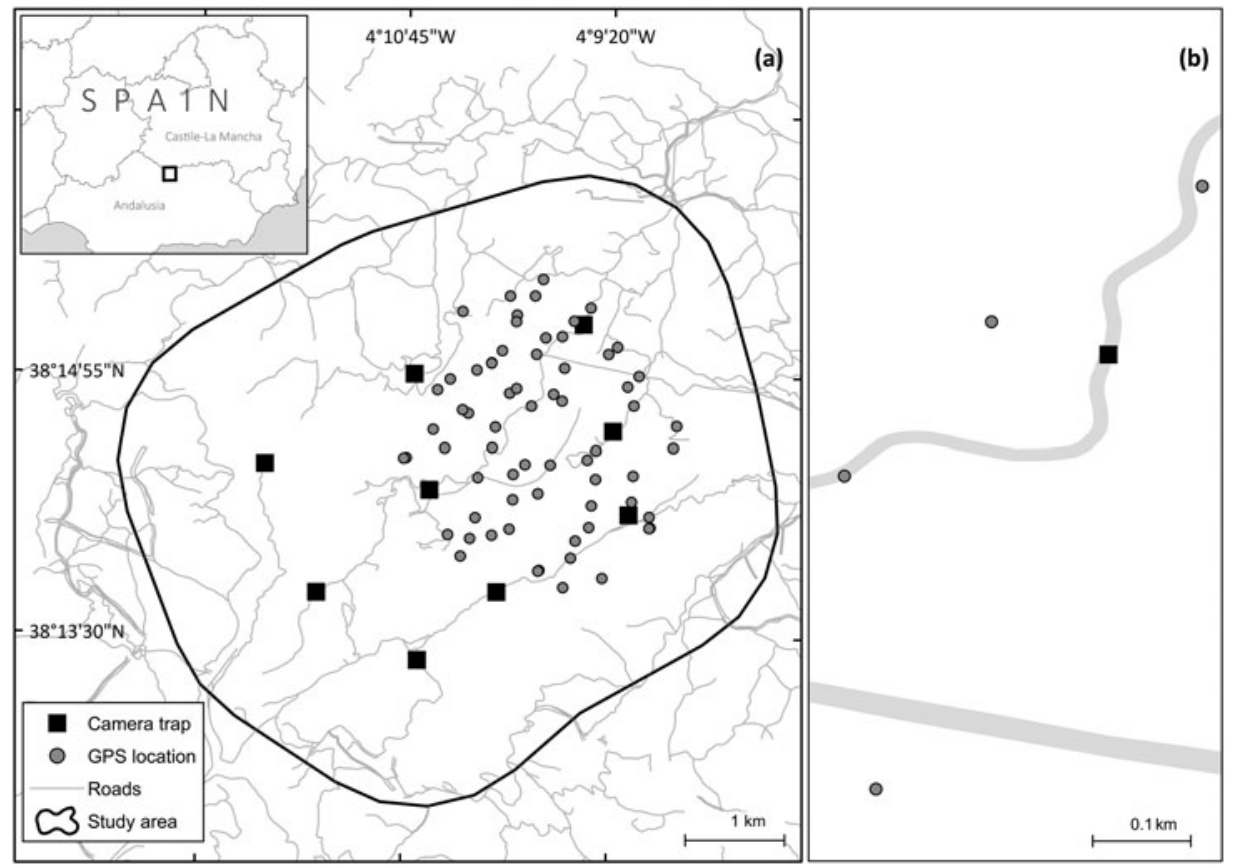

Fig. 1 (a) Location of the study area in southern Spain, design of the camera-trap grid, and locations obtained from GPS collar data for one Iberian lynx Lynx pardinus. (b) Detail of on-road and off-road Iberian lynx localities. distribution of home range centres in the study; Borchers \& Efford, 2008), and the detection function as half-normal (the capture probabilities decrease linearly with the distance of the camera station from the centre of the individual's home range; Efford et al., 2009).

Following Jůnek et al. (2015) we employed two models: the null model, in which detection is affected only by the use of space, and the 2-class finite mixture model, which allows for the modelling of variation in detection probability amongst individuals. We ranked candidate models using the Akaike information criterion corrected for small samples sizes (AICc). This analysis was performed using Density 5.0 (Efford \& Fewster, 2013).

\section{Random encounter model parameterization}

To convert camera-trapping rates into lynx density, we used the equation:

$$
D=\frac{y}{t} \frac{\pi}{v r(2+\theta)}
$$

following Rowcliffe et al. (2008), where $y$ is the number of independent photographic events, $t$ the total camera survey effort, $v$ the average speed of animal movement, and $r$ and $\theta$ the radius and angle of the camera trap detection zone, respectively.

We measured the $\operatorname{arc} \theta$ of the cameras through trials in which we placed a scale with $1-\mathrm{cm}$ divisions that could be read in photographs at a distance of $1.5 \mathrm{~m}$ from the camera. We activated the camera by walking in front of it and then measured the width of the photograph with the scale. We used trigonometry to infer the angle of the width of the photograph and the distance from the scale. We found that the camera radius was $5.5 \mathrm{~m}$, i.e. the other side of the track, thus reducing the possibility of an error associated with variation in the radius. We assumed the mean speed of movement of the Iberian lynx to be $8 \pm \mathrm{SE} 0.53 \mathrm{~km} /$ day, following Palomares et al. (2001).

We computed the overall variance in the random encounter model density estimates using bootstrapping, which incorporated the variance associated with the encounter rate (estimated by re-sampling camera locations with 10,000 replicas; Rowcliffe et al., 2008; Cusack et al., 2015a,b) and the standard errors associated with the estimation of the speed parameter. Camera-related parameters (Rowcliffe et al., 2008) were assumed to have no variance (Zero et al., 2013).

\section{Correction factor}

The correction factor represents the lynxes' preference for or avoidance of roads and is calculated as the ratio of road availability to road use in the study area (Fig. 1). The proportional availability of roads was defined by the surface area occupied by roads divided by the total size of the study area. To estimate the surface area occupied by roads we added a buffer of $2.25 \mathrm{~m}$ either side of the lines representing roads, resulting in a total width of $5.5 \mathrm{~m}$ (equal to the camera-trap detection radius). The total size of the study area, or effective trapping area, was defined as the minimum convex polygon around camera-trap stations with an added buffer strip of the mean maximum distance moved by recaptured lynx (Parmenter et al., 2003). To estimate the lynxes' use of roads, we used data from the only lynx in the study area equipped with a GPS collar, which gives a position signal every 4 hours and has an activity sensor. 
We used GPS data from the same months as the camera survey, but from the year following the camera-trapping surveys (September-October 2016). We only used locations recorded while the animal was active because the animal has to be moving to be photo-trapped. With these data, we determined and quantified the on-road and off-road localizations. The proportional use of roads by lynxes was defined as the number of on-road localizations out of all localizations. We conducted all spatial analyses with the geographical information system QGIS 2.18 (QGIS Development Team, 2014). We calculated the correction factor $\mathrm{CF}$ using the following equation:

$$
\mathrm{CF}=\frac{\text { road availability }}{\text { road use }}=\frac{\frac{\text { road surface }}{\text { total surface }}}{\frac{\text { on-road localizations }}{\text { total localizations }}}
$$

This factor corrects the random encounter model results (REM) and gives the corrected random encounter model result (REMc) as:

$$
\mathrm{REMc}=\mathrm{REM} \times \mathrm{CF}
$$

We used bootstrapping to assign measures of accuracy to the correction factor (re-sampling of the on-road and offroad locations with replacements) and then calculated the overall standard deviation and $95 \%$ confidence intervals for corrected random encounter model estimates, as described above. We carried out all analyses in $R$ 3.4.3 (R Development Core Team, 2017). As with the random encounter model estimates, we generated two corrected random encounter model estimates.

\section{Results}

Camera traps accumulated 567 trap days over a period of 63 days and generated 28 Iberian lynx detections, resulting in a total trapping rate of 0.93 captures/10o trap days. We identified all 10 lynxes previously known to inhabit the area; 387 trap days (43 days) were needed to capture all of them. The mean maximum distance moved was $1,494 \pm \mathrm{SE}$ $524 \mathrm{~m}$, which implies a total size of the study area of $3,027 \mathrm{ha}$.

Spatially explicitcapture-recapture surveys The null model $(\mathrm{AICc}=321.173 ; \Delta \mathrm{AIC}=0 ; 3$ parameters $)$ performed better than the 2-class finite mixture model $(\mathrm{AICc}=339.492$; $\Delta \mathrm{AIC}=14.31 ; 5$ parameters) and was used for the spatially explicit capture-recapture analysis. The population density estimates calculated using this model were $0.31 \pm \mathrm{SE} 0.13$ individuals/10o ha (Table 1). Movement parameter $\sigma$ was $991.54 \pm$ SE $228.97 \mathrm{~m}$.

Random encounter model The mean trapping rate in the dataset for the camera traps was $5.46 \pm$ SE 1.37 detections/ 100 trap days, resulting in a population density estimate of
TABLE 1 Density estimates $(D)$ for a population of 10 Iberian lynxes Lynx pardinus, obtained using different methodologies.

\begin{tabular}{ll}
\hline Method & $D \pm$ SE (95\% CI) \\
\hline Spatially explicit capture-recapture & $0.31 \pm 0.13(0.13-0.68)$ \\
Random encounter model & $1.43 \pm 0.34(0.42-3.74)$ \\
Corrected random encounter model & $0.36 \pm 0.21(0.10-0.94)$ \\
\hline
\end{tabular}

$1.43 \pm \mathrm{SE} 0.32$ individuals $/ 100$ ha based on the random encounter model.

Random encounter model with correction factor The total road surface area was 122.5 ha out of a total area of $3,027.7$ ha, which corresponds to a road availability of $4.05 \%$. We recorded 81 GPS localizations for lynx on the move, of which 13 were along roads; lynx road use was thus $16.07 \%$. Consequently the correction factor value was $0.25 \pm$ SE 0.08 and the estimated $95 \%$ confidence interval was $0.16-0.46$. After applying the correction factor to the random encounter model estimates, we obtained a density of $0.36 \pm$ SE 0.21 individuals/10o ha (Table 1). The random encounter model without the correction factor overestimates the lynx density by $378 \%$. The corrected random encounter model greatly improves the estimate as it only differs by $16 \%$ from the estimate calculated using the spatially explicit capture-recapture null model.

\section{Discussion}

Density estimates calculated with a random encounter model, using data from conventional camera-trapping surveys in which cameras are placed along tracks, resulted in an overestimate of lynx density in our study area. This was expected because the sampling design did not fulfil the key assumption that cameras should be placed randomly in relation to animal movements (Rowcliffe et al., 2013). Our results suggest that the application of a correction factor to the random encounter model based on lynx road use greatly improves the estimates. In addition, it allows us to use data from conventional survey designs in which cameras are positioned specifically to increase the capture probability; thus, the required sampling effort is less than in the standard random encounter model methodology with random camera placement. The random encounter model requires that cameras should be deployed for as long as is necessary to obtain a minimum of 10 photographs but preferably at least 20 (Rowcliffe et al., 2008). In Rovero et al. (2013) there is a personal communication from Rowcliffe that suggests the minimum number of photographs should be at least 50 . There are numerous studies of on-road vs off-road capture rates that provide an indication of the sampling effort needed to reach this number of captures. For instance, Di Bitetti et al. (2014) and Blake \& Mosquera (2014) have determined the on-road and off-road encounter rates for 
several mammal species. For the ocelot Leopardus pardalis (similar in size to the Iberian lynx), capture rates are 0.390.53 captures/10o trap days off-road vs $1.77-17.10$ captures/ 100 trap days on-road. These capture rates imply that 2,545-5,090 trapping days off-road and 59-566 trapping days on-road are required to obtain at least 10 captures. The effort required off-road is thus 5-32 times greater than on-road to achieve the same number of captures.

However, there are variations between carnivore species in the detection probability along tracks vs random camera placements. Although detection probability is greater along roads and main tracks for medium-sized and large carnivores (e.g. jaguars and ocelots), detection probabilities for small carnivores (e.g. Cape grey mongoose Galerella pulverulenta and common genet Genetta genetta) tend to be the same or greater with random camera placements (Wearn et al., 2013; Di Bitetti et al., 2014; Mann et al., 2015).

Photo capture rates are influenced by habitat structure and type, as well as by season (Larrucea et al., 2007; Harmsen et al., 2009; Cusack et al., 2015a,b; Mann et al., 2015). For example, tracks in dense vegetation possess a greater capacity for funnelling animals past a camera than tracks in open areas where animals have more room to wander off-track (Harmsen et al., 2009). Reproduction status (in those species where it is seasonal) may also influence the capture rate as it determines the movement range and thus the capture probability (Nichols et al., 1994). Male and female road use could also be different (Conde et al., 2010) and individual differences may also occur. In our case the correction factor was calculated using data from a single male Iberian lynx. The inclusion of more individuals of both sexes will improve the correction factor and thus the estimates obtained. However, the use of GPS collars on a sufficient number of individuals as a means of establishing a correction factor for different species will often be logistically and economically unviable. Thus, it would be advisable to examine the possibility of establishing the correction factor for unmarked species using on-road/off-road camera trap surveys. Additionally, the identification of correction factors for different species could make it possible to retrospectively estimate abundance using previously collected camera-trap data.

Although the use of roads is not as pronounced in herbivores as it is in carnivores, it has been observed in species such as the South American tapir Tapirus terrestris (Di Bitetti et al., 2014), Kirk's dik-dik Madoqua kirkii and the hippopotamus Hippopotamus amphibius (Cusack et al., 2015a,b). The estimates of herbivore densities established using a random encounter model (Zero et al., 2013; Caravaggi et al., 2016) do not take into account the possibility that species prefer to move along roads, which could affect results. It is thus important to carry out detailed studies of on-road vs off-road use by species for which individuals cannot be identified, to establish whether or not a correction factor needs to be applied when estimating population densities.
The application of the proposed correction factor to the random encounter model using conventional capturerecapture camera-trap methodology improves the accuracy and precision of lynx density estimates. The use of conventional camera-trap design data vs random-design data allows population estimates to be performed over shorter study periods, thereby reducing project costs (White, 1982; Garrote et al., 2012). Even though further studies (e.g. on habitat influence and intersexual variation) are needed to allow the correction factor to be used in other areas or species, the corrected random encounter model shows potential for estimating the density of species for which individual identification is not possible.

Acknowledgements The study was supported by LIFE Project 10NAT/ES/570 (Recovery of the historical distribution of the Iberian lynx (Lynx pardinus) in Spain and Portugal). We thank Pedro Monterroso and Sonia Illanas for their collaboration.

Author contributions Study conception and design: GG; data collection: GG, JMS, MR, SdL; data analysis: GG, RPdA, AA, MAS; writing: all authors.

\section{Conflict of interest None.}

Ethical standards This research abided by the Oryx guidelines on ethical standards. All necessary permits were obtained from Consejería de Medio Ambiente y Ordenación del Territorio (Andalusian government).

\section{References}

Anile, S., Ragni, B., Randi, E., Mattucci, F. \& Rovero, F. (2014) Wildcat population density on the Etna volcano, Italy: a comparison of density estimation methods. Journal of Zoology, 293, 252-261.

Balestrieri, A., Aritz Ruiz-González, A., Vergara, M., Capelli, E., Tirozzi, P., Alfino, S. et al. (2016) Pine marten density in lowland riparian woods: a test of the random encounter model based on genetic data. Mammalian Biology, $81,439-446$

Blake, J.G. \& Mosquera, D. (2014) Camera trapping on and off trails in lowland forest of eastern Ecuador: does location matter? Mastozoología Neotropical, 21, 17-26.

Borchers, D.L. \& EFFord, M.G. (2008) Spatially explicit maximum likelihood methods for capture-recapture studies. Biometrics, $64,377-385$.

Boron, V., Tzanopoulos, J., Gallo, J., Barragan, J., JaimesRodriguez, L., Schaller, G. \& Payán, E. (2016) Jaguar densities across human-dominated landscapes in Colombia: the contribution of unprotected areas to long term conservation. PLOS ONE, 11, e0153973.

Caravaggi, A., Zaccaroni, M., Riga, F., Schai-Braun, S.C., Dick, J.T., Montgomery, W.I. \& Reid, N. (2016) An invasivenative mammalian species replacement process captured by camera trap survey random encounter models. Remote Sensing in Ecology and Conservation, 2, 45-58.

Carbone, C., Christie, S., Conforti, K., Coulson, T., Franklin, N., Ginsberg, J.R. et al. (2001) The use of photographic rates to estimate densities of tigers and other cryptic mammals. Animal Conservation Forum, 4, 75-79. 
Conde, D.A., Colchero, F., Zarza, H., Christensen, N.L., Sexton, J.O., Manterola, C. et al. (2010) Sex matters: modeling male and female habitat differences for jaguar conservation. Biological Conservation, 143, 1980-1988.

Cusack, J.J., Dickman, A.J., Rowcliffe, J.M., Carbone, C. Macdonald, D.W. \& Coulson, T. (2015a) Random versus game trail-based camera trap placement strategy for monitoring terrestrial mammal communities. PLOS ONE, 10, e0126373.

Cusack, J.J., Swanson, A., Coulson, T., Packer, C., Carbone, C., DiCKMAN, A.J. et al. (2015b) Applying a random encounter model to estimate lion density from camera traps in Serengeti National Park, Tanzania: density estimation of Serengeti lions. The Journal of Wildlife Management, 79, 1014-1021.

Cutler, T.L. \& SWAnn, D.E. (1999) Using remote photography in wildlife ecology: a review. Wildlife Society Bulletin (1973-2006), 27, 571-581.

Di Bitetti, M.S., Paviolo, A. \& De Angelo, C. (2014) Camera trap photographic rates on roads vs. off roads: location does matter. Mastozoología Neotropical, 21, 37-46.

Efford, M.G. \& Fewster, R.M. (2013) Estimating population size by spatially explicit capture-recapture. Oikos, 122, 918-928.

Efford, M.G., Dawson, D.K. \& Borchers, D.L. (2009) Population density estimated from locations of individuals on a passive detector array. Ecology, 90, 2676-2682.

Foster, R.J. \& HARMSen, B.J. (2012) A critique of density estimation from camera-trap data. The Journal of Wildlife Management, $76,224-236$

Garrote, G., Perez de Ayala, R., Pereira, P., Robles, F., Guzman, N., García, F.J. et al. (2011) Estimation of the Iberian lynx (Lynx pardinus) population in the Doñana area, SW Spain, using capture-recapture analysis of camera-trapping data. European Journal of Wildlife Research, 57, 355-362.

Garrote, G., Gil-Sánchez, J.M., McCain, E.B., de Lillo, S., Tellería, J.L. \& Simón, M.Á. (2012) The effect of attractant lures in camera trapping: a case study of population estimates for the Iberian lynx (Lynx pardinus). European Journal of Wildlife Research, $58,881-884$.

Gil-Sánchez, J.M., Moral, M., Bueno, J., Rodríguez-Siles, J., Lillo, S., Pérez, J. et al. (2011) The use of camera trapping for estimating Iberian lynx (Lynx pardinus) home ranges. European Journal of Wildlife Research, 57, 1203-1211.

Harmsen, B.J., Foster, R.J., Silver, S.C., Ostro, L.E. \& Doncaster, C.P. (2009) Spatial and temporal interactions of sympatric jaguars (Panthera onca) and pumas (Puma concolor) in a neotropical forest. Journal of Mammalogy, 90, 612-620.

Jennelle, C.S., Runge, M.C. \& MacKenzie, D.I. (2002) The use of photographic rates to estimate densities of tigers and other cryptic mammals: a comment on misleading conclusions. Animal Conservation Forum, 5, 119-120.

Jưnek, T., Jủnková Vymyslická, P., Vymyslická, P.J., Hozdecká, K. \& Hejcmanová, P. (2015) Application of spatial and closed capture-recapture models on known population of the western derby eland (Taurotragus derbianus derbianus) in Senegal. PLOS ONE 10, e0136525.

Karanth, K.U. \& Nichols, J.D. (1998) Estimation of tiger densities in India using photographic captures and recaptures. Ecology, 79, 2852-2862.

Karanth, K.U., Nichols, J.D., Seidenstricker, J., Dinerstein, E., Smith, J.L.D., McDougal, C. et al. (2003) Science deficiency in conservation practice: the monitoring of tiger populations in India. Animal Conservation, 6, 141-146.

Larrucea, E.S., Brussard, P.F., Jaeger, M.M. \& Barrett, R.H. (2007) Cameras, coyotes, and the assumption of equal detectability. Journal of Wildlife Management, 71, 1682-1689.
Mann, G.K.H., O’Riain, M.J. \& Parker, D.M. (2015) The road less travelled: assessing variation in mammal detection probabilities with camera traps in a semi-arid biodiversity hotspot. Biodiversity and Conservation, 24, 531-545.

Nichols, J.D., Hines, J.E., Pollock, K.H., Hinz, R.L. \& Link, W.A. (1994) Estimating breeding proportions and testing hypotheses about costs of reproduction with capture-recapture data. Ecology, 75, 2052-2065.

O’Brien, T.G., Kinnaird, M.F. \& Wibisono, H.T. (2003) Crouching tigers, hidden prey: sumatran tiger and prey populations in a tropical forest landscape. Animal Conservation, 6, 131-139.

O’Connell, A.F. \& Nichols, J.D. (eds) (2011) Camera Traps in Animal Ecology: Methods and Analyses. Springer, Tokyo, Japan.

Palomares, F., Delibes, M., Revilla, E., Calzada, J. \& Fedriani, J.M. (2001) Spatial ecology of Iberian lynx and abundance of European rabbits in Southwestern Spain. Wildlife Monographs, $148,1-36$.

Parmenter, R.R., Yates, T.L., Anderson, D.R., Burnham, K.P., Dunnum, J.L., Franklin, A.B. et al. (2003) Small-mammal density estimation: a field comparison of grid-based vs. web-based density estimators. Ecological Monographs, 73, 1-26.

QGIS Development Team (2014) QGIS Geographic Information System. Open Source Geospatial Foundation Project. www.qgis.org/ en/site [accessed 25 June 2017].

R Development Core Team (2017) The R Project for Statistical Computing. www.r-project.org [accessed 20 December 2017].

Rovero, F. \& Marshall, A.R. (2009) Camera trapping photographic rate as an index of density in forest ungulates. Journal of Applied Ecology, 46, 1011-1017.

Rovero, F., Zimmermann, F., Berzi, D. \& Meek, P. (2013) 'Which camera trap type and how many do I need?' A review of camera features and study designs for a range of wildlife research applications. Hystrix, 24, 148-156.

Rowcliffe, J.M., Field, J., Turvey, S.T. \& Carbone, C. (2008) Estimating animal density using camera traps without the need for individual recognition. Journal of Applied Ecology, 45, 1228-1236.

Rowcliffe, J.M., Kays, R., Carbone, C. \& Jansen, P.A. (2013) Clarifying assumptions behind the estimation of animal density from camera trap rates: density estimates from camera trap rates. The Journal of Wildlife Management, 77, 876-876.

Simón, M.A., Gil-Sánchez, J.M., Ruiz, G., Garrote, G., Mccain, E., FernándeZ, L. et al. (2012) Reverse of the decline of the endangered Iberian Lynx. Conservation Biology, 26, $731-736$.

Trolle, M. \& Kéry, M. (2005) Camera-trap study of ocelot and other secretive mammals in the northern Pantanal. Mammalia, 69, 409-416.

Villette, P., Krebs, C.J., Jung, T.S. \& Boonstra, R. (2016) Can camera trapping provide accurate estimates of small mammal (Myodes rutilus and Peromyscus maniculatus) density in the boreal forest? Journal of Mammalogy, 97, 32-40.

Wearn, O.R., Rowcliffe, J.M., Carbone, C., Bernard, H. \& EwErs, R.M. (2013) Assessing the status of wild felids in a highly-disturbed commercial forest reserve in Borneo and the implications for camera trap survey design. PLOS ONE, 8, e77598.

White, G.C. (1982) Capture-Recapture and Removal Methods for Sampling Closed Populations. Los Alamos National Laboratory, Los Alamos, USA.

Zero, V.H., Sundaresan, S.R., O'Brien, T.G. \& Kinnaird, M.F. (2013) Monitoring an Endangered savannah ungulate, Grevy's zebra Equus grevyi: choosing a method for estimating population densities. Oryx, 47, 410-419. 